\title{
FUNCTIONS COMPLETELY MONOTONIC WITH RESPECT TO A SEQUENCE ${ }^{1}$
}

\author{
A. O. GARDER, JR.
}

The concept of completely monotonic functions was introduced by S. Bernstein in 1914. A function $f(x)$ is said to be completely monotonic in $a<x<b$ if and only if

$$
(-1)^{(k)} f^{(k)}(x) \geqq 0, \quad a<x<b, k=0,1,2, \cdots .
$$

In 1928 Bernstein established that complete monotonicity of $f(x)$ for $0 \leqq x<\infty$ is a necessary and sufficient condition that $f(x)$ have a representation

$$
f(x)=\int_{0}^{\infty} e^{-x t} d \alpha(t), \quad 0 \leqq x<\infty,
$$

as a Laplace-Stieltjes transform, where $\alpha(t)$ is bounded and nondecreasing $[4,144-163] .^{2}$

In 1949 Hirschman and Widder $[2,87,92,96]$ generalized Bernstein's theorem into a representation theory for the class of convolution transforms with totally positive kernels. Such a kernel is constructed as follows: Let $\left\{a_{n}\right\}_{n=1}^{\infty}$ be a sequence of nonzero real numbers such that $\sum_{n=1}^{\infty} a_{n}^{-2}<\infty$. Let $s$ be the complex variable $s=\sigma+i \tau$, and define the entire function

$$
E(s)=\prod_{n=1}^{\infty}\left[1-\frac{s}{a_{n}}\right] e^{s / a_{n}} .
$$

The totally positive kernel $G(t)$ is defined by

$$
G(t)=\frac{1}{2 \pi i} \int_{-i \infty}^{i \infty} \frac{e^{s t}}{E(s)} d s .
$$

Hirschman and Widder showed that a function $f(x)$ can be represented as a convolution transform

$$
f(x)=\int_{-\infty}^{\infty} G(x-t) d \beta(t), \quad \gamma<x<\infty,
$$

Received by the editors November 3, 1954 and, in revised form, January 21, 1955.

1 Presented to the Graduate School of Washington University in partial fulfillment of the requirements for the degree of Doctor of Philosophy. The author is indebted to Professor I. I. Hirschman for his encouragement and valuable direction.

2 The first number in brackets refers to the references at the end of the paper; the remaining numbers, to the pages. 
where $\beta(t)$ is nondecreasing and $\gamma$ is the abscissa of convergence of the transform $[1,182]$, if and only if (in case $a_{k}>0$ )

$$
f(x)=o\left(\exp \alpha_{2} x\right),
$$$$
x \rightarrow \infty,
$$

where $\alpha_{2}=\min _{k>0}\left(a_{k}\right)$, and, setting $D f(x)=f^{\prime}(x)$,

$$
g_{k}(x)=\prod_{n=1}^{k}\left(1-\frac{D}{a_{n}}\right) f(x) \geqq 0, \quad \gamma<x<\infty, k=0,1,2, \cdots
$$

Bernstein introduced a second definition of completely monotonic functions in terms of finite differences and proved that the class of functions so defined is equivalent to the class defined by (1). Using this second definition, he was able to show that, if a sequence of functions, each element of which can be represented as a Laplace transform of the form (2) in $0 \leqq x<\infty$, converges to a limiting function, then the limit can also be represented as a Laplace transform of the same type.

In the present paper, we shall proceed in a similar fashion to extend the representation theory for the transform (3).

Let $\left\{a_{k}\right\}_{k=1}^{\infty}$ be a sequence of nonzero real numbers with the property that

$$
\lim _{x \rightarrow \infty}\left|a_{k}\right|=\infty .
$$

Let $\left\{A_{k}\right\}_{k=-m, k \neq 0}^{n}$ be any selection from this sequence satisfying

$$
\begin{array}{r}
A_{-m}<A_{-(m-1)}<\cdots<A_{-1}<0<A_{1}<A_{2}<\cdots<A_{n}, \\
m, n=0,1,2, \cdots .
\end{array}
$$

We note that the range of $m$ or $n$ is bounded in case the sequence $\left\{a_{k}\right\}_{k=1}^{\infty}$ has a finite number of negative or of positive terms, respectively.

Definition 1 . The function $f(x)$ is said to be completely monotonic $(D, A)$ with respect to the sequence $\left\{a_{k}\right\}_{k=1}^{\infty}$ in the interval $a<x<b$ if and only if

$$
g_{m, n}(x)=\prod_{k=-m, k \neq 0}^{n}\left(1-\frac{D}{A_{k}}\right) f(x) \geqq 0, a<x<b, m, n=0,1,2, \cdots,
$$

for all selections $\left\{A_{k}\right\}_{k=-m, k \neq 0}^{n}$ from the sequence $\{a\}_{k=1}^{\infty}$ which satisfy (6). We shall write $g_{0, n}(x)=g_{n}(x)$ to agree with the notation introduced in inequality (4).

Definition 2. The function $f(x)$ is said to be completely monotonic $(\Delta, A)$ with respect to the sequence $\left\{a_{k}\right\}_{k-1}^{\infty}$ in the interval $a<x<b$ if and only if 


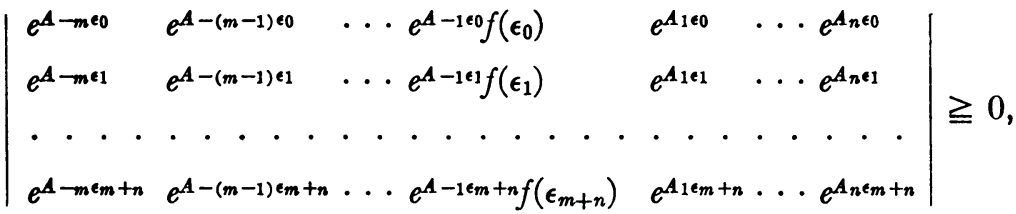

$$
\begin{aligned}
& m, n=0,1,2, \cdots
\end{aligned}
$$

for all $\left\{A_{k}\right\}_{k=-m, k \neq 0}^{n}$ satisfying (6) and all real numbers $\left\{\epsilon_{k}\right\}_{k=0}^{m+n}$ satisfying

$$
a<\epsilon_{0}<\epsilon_{1}<\cdots<\epsilon_{m+n}<b .
$$

The equivalence of these two definitions is contained in the following two theorems.

THEOREM 3. If the function $f(x)$ is completely monotonic $(\Delta, A)$ in $a<x<b$, then it is completely monotonic $(D, A)$ in $a<x<b$.

The proof of this theorem will follow the proof of

THEOREM 4. If $f(x)$ is completely monotonic $(D, A)$ in $a<x<b$, then it is completely monotonic $(\Delta, A)$ in $a<x<b$.

To prove this, we shall need a result $[3,49]$ which we state as

TheOREM 5. Let $\alpha_{1}<\alpha_{2}<\cdots<\alpha_{n}$. Let $\beta_{1}<\beta_{2}<\cdots<\beta_{n}$. Then the determinant

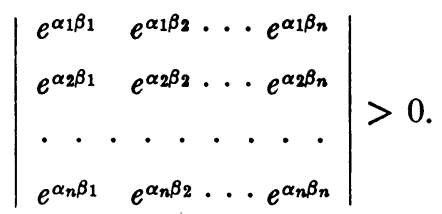

Proof of Theorem 4. Since

$$
\left(1-\frac{D}{A}\right) f(x)=\frac{e^{A x} D e^{-A x} f(x)}{-A},
$$

Rolle's theorem implies that, if $f(x)$ has $n$ zeros in $a<x<b$, then $(1-D / A) f(x)$ has at least $n-1$ zeros in that interval. We set

$$
F(x)=f(x)-\sum_{k=-m, k \neq 0}^{n} b_{k} e^{A_{k} x}-b_{0},
$$

where $\left\{b_{k}\right\}_{k=-m}^{n}$ are constants to be determined. It is clear that

$$
\prod_{k=m, k \neq 0}^{n}\left(1-\frac{D}{A_{k}}\right) F(x)=g_{m, n}(x)-b_{0} .
$$


Let $\epsilon_{0}, \epsilon_{1}, \cdots, \epsilon_{m+n}$ be any real numbers satisfying (7). By Theorem 5 we may choose $\left\{b_{k}\right\}_{k=-m}^{n}$ such that

$$
F\left(\epsilon_{i}\right)=0, \quad j=0,1, \cdots, m+n .
$$

Thus $F(x)$ has $m+n+1$ zeros, so that there exists $\epsilon, \epsilon_{0}<\epsilon<\epsilon_{n+m}$, for which

$$
\prod_{k=-m, k \neq 0}^{n}\left(1-\frac{D}{A_{k}}\right) F(\epsilon)=0 .
$$

Setting $x=\epsilon$ in (8), we obtain that

$$
b_{0}=g_{m, n}(\epsilon) \geqq 0 .
$$

From the definition of $b_{0}$ and from Theorem 5, it follows that the determinant

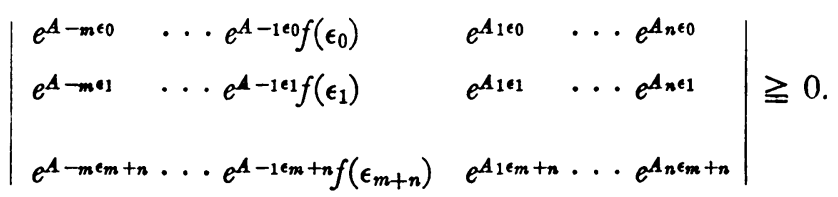

Thus $f(x)$ is completely monotonic $(\Delta, A)$.

Now we establish the converse, i.e., Theorem 3. For the purpose of simplification, we henceforth suppose that $f(x)$ is completely monotonic $(\Delta, A)$ in the interval $-\infty<x<\infty$. Further, we assert that it will be sufficient to consider sequences $\left\{a_{k}\right\}_{k=1}^{\infty}$ of positive terms. In other words, we need to show only that, if $f(x)$ is completely monotonic $(\Delta, A)$ with respect to a sequence $\left\{a_{k}\right\}_{k=1}^{\infty}$ of positive terms satisfying (5), then $f(x)$ is completely monotonic $(D, A)$. For, if this assertion has been proved, and if $h(x)$ is completely monotonic $(\Delta, A)$ with respect to a sequence of nonzero numbers satisfying (5), the following argument shows that $h(x) \in C^{\infty}$. Either the sequence contains a subsequence of positive terms or it contains a subsequence of negative terms, or both. In the first case, $h(x)$ is completely monotonic $(\Delta, A)$, and hence $(D, A)$, with respect to the positive subsequence; thus $h(x) \in C^{\infty}$. In the second case, making use of elementary properties of determinants, we may observe that $h(-x)$ is completely monotonic $(\Delta, A)$ with respect to the positive subsequence obtained by taking the negative of each term of the negative subsequence; thus we again have that $h(x) \in C^{\infty}$. We may then review the arguments in the proof of Theorem 4 to conclude that $h(x)$ is completely monotonic $(D, A)$.

We now proceed to the proof of Theorem 3 for the case in which $\left\{a_{k}\right\}_{k=1}^{\infty}$ is a sequence of positive real numbers satisfying (5). 
LeMma 6. Let $f(x)$ be completely monotonic $(\Delta, A)$. If we set $g(x)$ $=e^{-A_{1} x} f(x)$, then $g(x)$ is a non-negative, nonincreasing function of $x$.

Proof. Taking $n=0$ in Definition 2 , we see that $f(x) \geqq 0$; hence $g(x) \geqq 0$. Taking $n=1$, we have that

$$
\left|\begin{array}{ll}
f\left(\epsilon_{0}\right) & e^{A_{1} \epsilon_{0}} \\
f\left(\epsilon_{1}\right) & e^{A_{1 \epsilon_{1}}}
\end{array}\right| \geqq 0 .
$$

Hence $g\left(\epsilon_{0}\right) \geqq g\left(\epsilon_{1}\right)$.

Lemma 7. If $G(x)=g\left[(\log x) /\left(A_{2}-A_{1}\right)\right], G(x)$ is convex and continuous in the interval $0<x<\infty$. Hence the function $g(x)$, and thus $f(x)$, is continuous for $-\infty<x<\infty$.

Proof. Let $x_{0}<x_{1}<x_{2}$. Set $y_{i}=e^{\left(A_{2}-A_{1}\right) x_{i}}$ for $i=0,1,2$. Taking $n=2$ in Definition 2, we have that

$$
\left|\begin{array}{lll}
G\left(y_{0}\right) & 1 & y_{0} \\
G\left(y_{1}\right) & 1 & y_{1} \\
G\left(y_{2}\right) & 1 & y_{2}
\end{array}\right| \geqq 0 .
$$

From this it follows that $G(x)$ is convex. Since, from Lemma 6, $G(x)$ is nonincreasing, it follows that $G(x)$ is continuous.

Since $G(x)$ is a convex function, we know $[4,148-150]$ that $G_{L}^{\prime}(x)$ and $G_{R}^{\prime}(x)$, the left and right-hand derivatives of $G(x)$, exist and we have that

$$
G_{L}^{\prime}(x) \leqq G_{R}^{\prime}(x) \leqq 0, \quad 0<x<\infty .
$$

Thus we deduce

Corollary 8. $g_{L}^{\prime}(x)$ and $g_{R}^{\prime}(x)$, the left and right-hand derivatives of $g(x)$, exist. If $x<y$, we have that

$$
g_{L}^{\prime}(x) \leqq g_{R}^{\prime}(x) \leqq g_{L}^{\prime}(y), \quad-\infty<x<y<\infty .
$$

LEMMA 9. If we set $F(x)=f(x)-\left[f_{L}^{\prime}(x) / A_{1}\right]$, we have that

$$
F(x)=-\frac{e^{A_{1} x}}{A_{1}} g_{L}^{\prime}(x) .
$$

Lemma 10. Delete from the sequence $\left\{a_{k}\right\}_{k=1}^{\infty}$ all terms which are less than or equal to $A_{1}$. Designate the new sequence by $A^{\prime}$. The function $F(x)$ of Lemma 9 is completely monotonic $\left(\Delta, A^{\prime}\right)$. In other words, let $A_{2}<\cdots<A_{n}$ be an arbitrary selection from the sequence $A^{\prime}$; let $\left\{\epsilon_{k}\right\}_{k=0}^{n-1}$ satisfy $\epsilon_{0}<\epsilon_{1}<\cdots<\epsilon_{n-1} ;$ then 


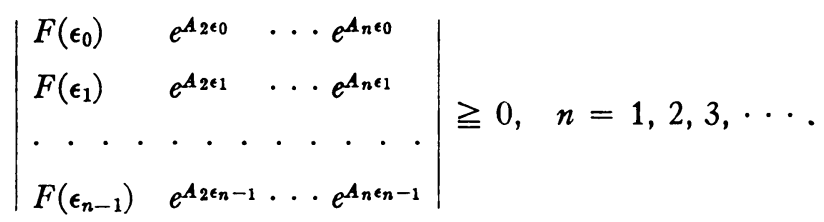

Before proving Lemma 10 , we observe that it may be used to complete the proof of Theorem 3. Lemma 7 established that, if a function is completely monotonic $(\Delta, A)$, then it is continuous. Hence it follows from Lemma 10 that $F(x)$, and thus $g_{L}^{\prime}(x)$, is continuous (Lemma 9). But from Corollary 8, this implies that the continuous function $g(x)$ has a derivative $g^{\prime}(x)$ at every point. Thus $f^{\prime}(x)$ exists and $f^{\prime}(x)=f_{L}^{\prime}(x)$. If we take $n=1$ in Lemma 10 we have that

$$
F(x)=f(x)-\frac{f_{L}^{\prime}(x)}{A_{1}}=\left(1-\frac{D}{A_{1}}\right) f(x) \geqq 0 .
$$

But this establishes that, if a function $f(x)$ is completely monotonic $(\Delta, A)$, then $\left(1-D / A_{1}\right) f(x) \geqq 0$. But by Lemma 10 , the latter function is itself completely monotonic $\left(\Delta, A^{\prime}\right)$ so that

$$
\left(1-\frac{D}{A_{1}}\right)\left(1-\frac{D}{A_{2}}\right) f(x) \geqq 0 .
$$

Repeated applications of Lemma 10 then give

$$
\prod_{k=1}^{n}\left(1-\frac{D}{A_{k}}\right) f(x) \geqq 0 \text {, }
$$

or, $f(x)$ is completely monotonic $(D, A)$, which completes the proof of Theorem 3.

We now prove Lemma 10.

For the case $n=1$, we must show that $F(x) \geqq 0$. But since $f(x)$ is completely monotonic $(\Delta, A)$, we have that, for $\delta>0$ and any $x$,

$$
\left|\begin{array}{cc}
g(x-\delta) & 1 \\
g(x) & 1
\end{array}\right| \geqq 0
$$

or

$$
(g(x-\delta)-g(x)) /-\delta \leqq 0 .
$$

Letting $\delta$ tend to zero, this implies that $g_{L}^{\prime}(x) \leqq 0$, which in turn implies, by Lemma 9 , that $F(x) \geqq 0$.

For the case $n=2$, we write $\Delta_{2}=A_{2}-A_{1}$, and we have that, for $\delta>0$, 


$$
\left|\begin{array}{lll}
g\left(\epsilon_{0}-\delta\right) & 1 & e^{\Delta_{\mathbf{2}}\left(\epsilon_{0}-\delta\right)} \\
g\left(\epsilon_{0}\right) & 1 & e^{\Delta_{\mathbf{2} \epsilon_{0}}} \\
g\left(\epsilon_{1}\right) & 1 & e^{\Delta_{2 \epsilon_{1}}}
\end{array}\right| \geqq 0
$$

and also that, for $\delta$ sufficiently small,

$$
\left|\begin{array}{lll}
g\left(\epsilon_{0}\right) & 1 & e^{\Delta_{2 \epsilon_{0}}} \\
g\left(\epsilon_{1}-\delta\right) & 1 & e^{\Delta_{2}\left(\epsilon_{1}-\delta\right)} \\
g\left(\epsilon_{1}\right) & 1 & e^{\Delta_{2 \epsilon_{1}}}
\end{array}\right| \geqq 0
$$

We conclude from (9) that the second order determinant

$$
\left|\begin{array}{ll}
g\left(\epsilon_{0}-\delta\right)-g\left(\epsilon_{0}\right) & e^{\epsilon_{0} \Delta_{2}}\left[e^{-\delta \Delta_{2}}-1\right] \\
g\left(\epsilon_{1}\right)-g\left(\epsilon_{0}\right) & e^{\epsilon_{1} \Delta_{2}}-e^{\epsilon_{0} \Delta_{2}}
\end{array}\right| \geqq 0 .
$$

Hence, multiplying the first row by $-1 / \delta$, and letting $\delta$ tend to zero, we obtain

$$
\left|\begin{array}{ll}
g_{L}^{\prime}\left(\epsilon_{0}\right) & \Delta_{2} e^{\epsilon_{0} \Delta_{2}} \\
g\left(\epsilon_{1}\right)-g\left(\epsilon_{0}\right) & e^{\epsilon_{1} \Delta_{2}}-e^{\epsilon_{0} \Delta_{2}}
\end{array}\right| \leqq 0 .
$$

Now apply Lemma 9 to obtain the inequality

$$
\left|\begin{array}{ll}
F\left(\epsilon_{0}\right) & -e^{\epsilon_{0} A_{2}} \\
g\left(\epsilon_{1}\right)-g\left(\epsilon_{0}\right) & \left(A_{1} / \Delta_{2}\right)\left[e^{\epsilon_{1} \Delta_{2}}-e^{\epsilon_{0} \Delta_{2}}\right]
\end{array}\right| \geqq 0 .
$$

Similarly, from (10) we obtain the inequality

$$
\left|\begin{array}{ll}
F\left(\epsilon_{1}\right) & -e^{\epsilon_{1} A_{2}} \\
g\left(\epsilon_{1}\right)-g\left(\epsilon_{0}\right) & \left(A_{1} / \Delta_{2}\right)\left[e^{\epsilon_{1} \Delta_{2}}-e^{\epsilon_{0} \Delta_{2}}\right]
\end{array}\right| \leqq 0 .
$$

Multiplying the first row of (11) by $e^{A_{2 \epsilon_{1}}}$, the first row of (12) by $-e^{A_{260}}$, we add the resulting inequalities to obtain

$$
\left|\begin{array}{lc}
F\left(\epsilon_{0}\right) e^{\epsilon_{1} A_{2}}-F\left(\epsilon_{1}\right) e^{\epsilon_{0} A_{2}} & 0 \\
g\left(\epsilon_{1}\right)-g\left(\epsilon_{0}\right) & \left(A_{1} / \Delta_{2}\right)\left[e^{\epsilon_{1} \Delta_{2}}-e^{\epsilon_{0} \Delta_{2}}\right]
\end{array}\right| \geqq 0 .
$$

But we see that the term in the first column and first row is precisely the desired determinant. Thus we deduce that

$$
\left|\begin{array}{ll}
F\left(\epsilon_{0}\right) & e^{A} \epsilon_{0} \\
F\left(\epsilon_{1}\right) & e^{A_{2} \epsilon_{1}}
\end{array}\right| \geqq 0 .
$$

Now we use Theorem 5 to show that this method generalizes to the case $n=3$; from this case it will be readily seen how to establish our result for arbitrary $n$. 
As before, since $f(x)$ is completely monotonic $(\Delta, A)$, if we choose $\delta>0$ sufficiently small, and if we write $\Delta_{2}=A_{2}-A_{1}, \Delta_{3}=A_{3}-A_{1}$, we have the inequalities

$$
\begin{aligned}
& \left|\begin{array}{llll}
g\left(\epsilon_{0}-\delta\right) & 1 & e^{\left(\epsilon_{0}-\delta\right) \Delta_{2}} & e^{\left(\epsilon_{0}-\delta\right) \Delta_{3}} \\
g\left(\epsilon_{0}\right) & 1 & e^{\epsilon_{0} \Delta_{2}} & e^{\epsilon_{0} \Delta_{3}} \\
g\left(\epsilon_{1}\right) & 1 & e^{\epsilon_{1} \Delta_{2}} & e^{\epsilon_{1} \Delta_{3}} \\
g\left(\epsilon_{2}\right) & 1 & e^{\epsilon_{2} \Delta_{2}} & e^{\epsilon_{2} \Delta_{3}}
\end{array}\right| \geqq 0, \\
& \left|\begin{array}{llll}
g\left(\epsilon_{0}\right) & 1 & e^{\epsilon_{0} \Delta_{2}} & e^{\epsilon_{0} \Delta_{3}} \\
g\left(\epsilon_{1}-\delta\right) & 1 & e^{\left(\epsilon_{1}-\delta\right) \Delta_{2}} & e^{\left(\epsilon_{1}-\delta\right) \Delta_{3}} \\
g\left(\epsilon_{1}\right) & 1 & e^{\epsilon_{1} \Delta_{2}} & e^{\epsilon_{1} \Delta_{3}} \\
g\left(\epsilon_{2}\right) & 1 & e^{\epsilon_{2} \Delta_{2}} & e^{\epsilon_{2} \Delta_{3}}
\end{array}\right| \geqq 0, \\
& \left|\begin{array}{llll}
g\left(\epsilon_{0}\right) & 1 & e^{\epsilon_{0} \Delta_{2}} & e^{\epsilon_{0} \Delta_{3}} \\
g\left(\epsilon_{1}\right) & 1 & e^{\epsilon_{1} \Delta_{2}} & e^{\epsilon_{1} \Delta_{3}} \\
g\left(\epsilon_{2}-\delta\right) & 1 & e^{\left(\epsilon_{2}-\delta\right) \Delta_{2}} & e^{\left(\epsilon_{2}-\delta\right) \Delta_{3}} \\
g\left(\epsilon_{2}\right) & 1 & e^{\epsilon_{2} \Delta_{2}} & e^{\epsilon_{2} \Delta_{3}}
\end{array}\right| \geqq 0 .
\end{aligned}
$$

We must show that the determinant

$$
F_{3}=\left|\begin{array}{lll}
F\left(\epsilon_{0}\right) & e^{\epsilon_{0} A_{2}} & e^{\epsilon_{0} A_{3}} \\
F\left(\epsilon_{1}\right) & e^{\epsilon_{1} A_{2}} & e^{\epsilon_{1} A_{3}} \\
F\left(\epsilon_{2}\right) & e^{\epsilon_{2} A_{2}} & e^{\epsilon_{2} A_{3}}
\end{array}\right| \geqq 0 .
$$

By calculations similar to those in the previous case, we obtain from inequality (13) that

$$
\left|\begin{array}{lll}
F\left(\epsilon_{0}\right) & -e^{\epsilon_{0} \Delta_{2}} & -e^{\epsilon_{0} \Delta_{3}} \\
g\left(\epsilon_{1}\right)-g\left(\epsilon_{0}\right) & \frac{A_{1}}{\Delta_{2}}\left[e^{\epsilon_{1} \Delta_{2}}-e^{\epsilon_{0} \Delta_{2}}\right] & \frac{A_{1}}{\Delta_{3}}\left[e^{\epsilon_{1} \Delta_{3}}-e^{\epsilon_{0} \Delta_{3}}\right] \\
g\left(\epsilon_{2}\right)-g\left(\epsilon_{0}\right) & \frac{A_{1}}{\Delta_{2}}\left[e^{\epsilon_{2} \Delta_{2}}-e^{\epsilon_{0} \Delta_{2}}\right] & \frac{A_{1}}{\Delta_{3}}\left[e^{\epsilon_{2} \Delta_{3}}-e^{\epsilon_{0} \Delta_{3}}\right]
\end{array}\right| \geqq 0 ;
$$

from inequality (14), that

$$
\left|\begin{array}{lll}
F\left(\epsilon_{1}\right) & -e^{\epsilon_{1} A_{2}} & -e^{\epsilon_{1} A_{3}} \\
g\left(\epsilon_{1}\right)-g\left(\epsilon_{0}\right) & \frac{A_{1}}{\Delta_{2}}\left[e^{\epsilon_{1} \Delta_{2}}-e^{\epsilon_{0} \Delta_{2}}\right] & \frac{A_{1}}{\Delta_{3}}\left[e^{\epsilon_{1} \Delta_{3}}-e^{\epsilon_{0} \Delta_{3}}\right] \\
g\left(\epsilon_{2}\right)-g\left(\epsilon_{0}\right) & \frac{A_{1}}{\Delta_{2}}\left[e^{\epsilon_{2} \Delta_{2}}-e^{\epsilon_{0} \Delta_{2}}\right] & \frac{A_{1}}{\Delta_{3}}\left[e^{\epsilon_{2} \Delta_{3}}-e^{\epsilon_{0} \Delta_{3}}\right]
\end{array}\right| \leqq 0 ;
$$


from inequality (15), that

$$
\left|\begin{array}{lll}
F\left(\epsilon_{2}\right) & -e^{\epsilon_{2} \Delta_{2}} & -e^{\epsilon_{2} \Delta_{3}} \\
g\left(\epsilon_{1}\right)-g\left(\epsilon_{0}\right) & \frac{A_{1}}{\Delta_{2}}\left[e^{\epsilon_{1} \Delta_{2}}-e^{\epsilon_{0} \Delta_{2}}\right] & \frac{A_{1}}{\Delta_{3}}\left[e^{\epsilon_{1} \Delta_{3}}-e^{\epsilon_{0} \Delta_{3}}\right] \\
g\left(\epsilon_{2}\right)-g\left(\epsilon_{0}\right) & \frac{A_{1}}{\Delta_{2}}\left[e^{\epsilon_{2} \Delta_{2}}-e^{\epsilon_{0} \Delta_{2}}\right] & \frac{A_{1}}{\Delta_{3}}\left[e^{\epsilon_{2} \Delta_{3}}-e^{\epsilon_{0} \Delta_{3}}\right]
\end{array}\right| \geqq 0 .
$$

Now multiply inequality (16) by the minor of $F\left(\epsilon_{0}\right)$ in the determinant $F_{3} ;(17)$ by the negative of the minor of $F\left(\epsilon_{1}\right)$, and (18) by the minor of $F\left(\epsilon_{2}\right)$. Observing that each of the three minors is positive by Theorem 5 , we add the three inequalities so obtained and we see that, after factoring positive constants from the second and third columns, the resulting inequality is

$$
\left|\begin{array}{ccc}
F_{3} & 0 & 0 \\
g\left(\epsilon_{1}\right)-g\left(\epsilon_{0}\right) & e^{\epsilon_{1} \Delta_{2}}-e^{\epsilon_{0} \Delta_{2}} & e^{\epsilon_{1} \Delta_{3}}-e^{\epsilon_{0} \Delta_{3}} \\
g\left(\epsilon_{2}\right)-g\left(\epsilon_{0}\right) & e^{\epsilon_{2} \Delta_{2}}-e^{\epsilon_{0} \Delta_{2}} & e^{\epsilon_{2} \Delta_{3}}-e^{\epsilon_{0} \Delta_{3}}
\end{array}\right| \geqq 0 .
$$

Thus $F_{3}$ is nonnegative, as desired, if its minor in the determinant on the left side of inequality (19) is positive. But that minor is

$$
e^{\epsilon_{0} \Delta_{2}} e^{\epsilon_{0} \Delta_{3}}\left|\begin{array}{ll}
e^{\left(\epsilon_{1}-\epsilon_{0}\right) \Delta_{2}}-1 & e^{\left(e_{1}-\epsilon_{0}\right) \Delta_{3}}-1 \\
e^{\left(\epsilon_{2}-\epsilon_{0}\right) \Delta_{2}}-1 & e^{\left(\epsilon_{2}-\epsilon_{0}\right) \Delta_{3}}-1
\end{array}\right|
$$

this is positive by the following result, which is easily deduced from Theorem 5:

Let

$$
0<\alpha_{1}<\alpha_{2}<\cdots<\alpha_{n}, \quad 0<\beta_{1}<\beta_{2}<\cdots<\beta_{n} .
$$

The determinant

$$
\left|\begin{array}{ccc}
e^{\alpha_{1} \beta_{1}}-1 & e^{\alpha_{1} \beta_{2}}-1 \cdots e^{\alpha_{1} \beta_{n}}-1 \\
e^{\alpha_{2} \beta_{1}}-1 & e^{\alpha_{2} \beta_{2}}-1 \cdots \cdot e^{\alpha_{2} \beta_{n}}-1 \\
\cdot \cdot \cdot \cdot & \cdot \cdot \cdot \cdot \cdot \cdot \cdot \cdot \cdot \cdot \cdot \\
e^{\alpha_{n} \beta_{1}}-1 & e^{\alpha_{n} \beta_{2}}-1 \cdots \cdot e^{\alpha_{n} \beta_{n}}-1
\end{array}\right|>0 .
$$

This completes the proof of Lemma 10.

THEOREM 11. Let $\left\{u_{n}(x)\right\}_{n=1}^{\infty}$ be a sequence of functions. Let each function of the sequence be completely monotonic $(A)$ in $-\infty<x<\infty$. Let the series 


$$
\sum_{n=1}^{\infty} u_{n}(x)
$$

converge to a function $f(x)$ in $-\infty<x<\infty$. Then $f(x)$ is completely monotonic $(A)$.

Proof. We have that

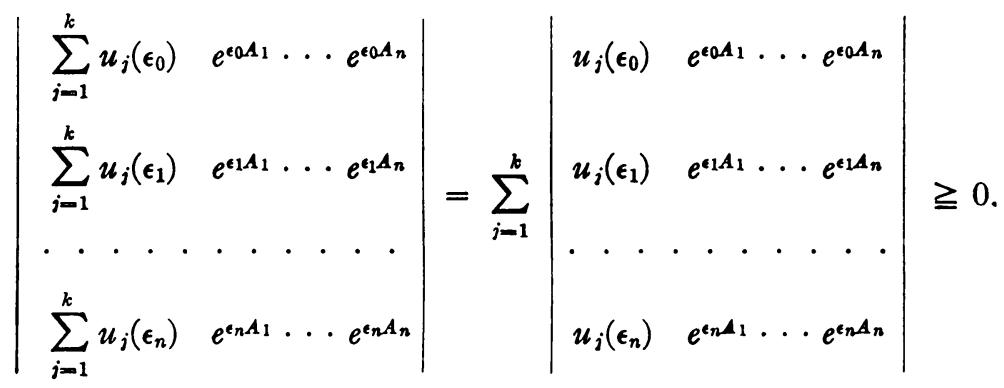

Let $k$ tend to $\infty$ to obtain the desired result.

ThEOREM 12. Let $\left\{u_{n}(x)\right\}_{n=1}^{\infty}$ be a sequence of functions which converge to a limit function $f(x)$. Let $u_{n}(x)$ be representable as a convolution transform with a totally positive kernel $G(t)$ constructed from $a_{k}>0$

$$
u_{n}(x)=\int_{-\infty}^{\infty} G(x-t) d \beta_{n}(t), \quad-\infty<x<\infty ; \quad n=1,2,3, \cdots,
$$

and let $f(x)=o\left(e^{\alpha_{2} x}\right)$ as $x \rightarrow \infty$. There exists a nondecreasing function $\beta(t)$ such that the transform

$$
\int_{-\infty}^{\infty} G(x-t) d \beta(t)
$$

converges to $f(x)$ in $-\infty<x<\infty$.

\section{BIBLIOGRAPHY}

1. I. I. Hirschman and D. V. Widder, The inversion of a general class of convolution transforms, Trans. Amer. Math. Soc. vol. 66 (1949) pp. 135-201.

2. - A representation theory for a general class of convolution transforms, Trans. Amer. Math. Soc. vol. 67 (1949) pp. 69-97.

3. G. Polya and G. Szegö, Aufgaben und Lehrsutze aus der Analysis, vol. 2, New York, 1925.

4. D. V. Widder, The Laplace transform, Princeton, 1946.

WASHINGTON UNIVERSITY 